13

\title{
Профильный анализ пленок ферритов-гранатов методом оптической эмиссионной спектроскопии тлеющего разряда
}

\author{
(C) А.А. Федоренко, В.Н. Бержанский, А.В. Каравайников, А.Н. Шапошников, А.Р. Прокопов \\ Крымский фредеральный университет им. В.И. Вернадского, \\ 295007 Симферополь, Россия \\ e-mail: shalex53@gmail.com
}

Поступило в Редакцию 24 мая 2020 г.

В окончательной редакции 19 августа 2020 г.

Принято к публикации 21 августа 2020 г.

\begin{abstract}
Представлены результаты экспериментов по применению метода оптической эмиссионной спектроскопии тлеющего разряда для профильного анализа вакуумно-осажденных одно- и двуслойных пленок многокомпонентных ферритов-гранатов различного состава до и после кристаллизационного отжига. Выявлено наличие внутренних и внешних переходных слоев, и исследована модификация их элементного состава при кристаллизации и различной последовательности синтеза отдельных компонент в феррит-гранатовых гетероструктурах.
\end{abstract}

Ключевые слова: оптическая эмиссионная спектроскопия тлеющего разряда, профильный анализ, пленки ферритов-гранатов.

DOI: 10.21883/JTF.2021.02.50372.187-20

\section{Введение}

Использование метода оптической эмиссионной спектроскопии тлеющего разряда (Glow Discharge Optical Emission Spectroscopy - GDOES) позволяет провести определение химического состава образца, выявить распределение элементов по толщине (профиль), исследовать границы раздела между покрытием и подложкой, определить их толщину и влияние различных воздействий на распределение элементов $[1,2]$.

Достаточно полно применение метода GDOES с демонстрацией его возможностей в плане разрешения и чувствительности представлено в ряде обзоров [3-6]. Например, профильный анализ нанокомпозитных покрытий $\mathrm{Ti}-\mathrm{Zr}-\mathrm{C}-\mathrm{O}-\mathrm{N}$ толщиной $10 \mathrm{~nm}$, нанесенных на монокристаллический кремний магнетронным распылением мишени $\mathrm{TiZrC}+\mathrm{ZrO}_{2}$, позволил уверенно выделить три зоны размером от 2 до $3 \mathrm{~nm} \mathrm{c} \mathrm{перепадом}$ концентраций отдельных элементов [6]. Исследованы различные двуслойные нанокомпозиты на основе $\mathrm{Ti}, \mathrm{Cr}$, Al, N, C [7-12]. В работе [13] представлены результаты профильного анализа последовательно нанесенных на Si-подложку 60 слоев TiN/TiAlN/. . .TiAlN/TiN с толщиной каждого из слоев $3 \mathrm{~nm}$. Степень обогащения медью интерфейсов анодированных пленок бинарных сплавов $\mathrm{Al}-\mathrm{Cu}$ в зависимости от технологических параметров процесса исследована в $[14,15]$. Применение GDOES для качественного и количественного анализа состава покрытий жестких магнитных дисков описано в [16,17].

Использование добавок $\mathrm{N}_{2}, \mathrm{O}_{2}, \mathrm{H}_{2}$ в количестве $0.5-10.0 \%$ к рабочему газу (аргону) позволяет благодаря улучшению формы кратера увеличить разрешение по глубине [18] и достичь рекордных значений разрешения по глубине (на уровне толщины атомных монослоев) [19].

Значительно меньше работ посвящено использованию метода GDOES для анализа диэлектрических покрытий. Характеризация поверхностей, интерфейсов и межфазных границ в таких материалах является сложной аналитической задачей. Скорости распыления в этом случае значительно снижены по сравнению с проводящими материалами ( $2 \mathrm{~nm} / \mathrm{s}$ для стекла по сравнению с $50-150 \mathrm{~nm} / \mathrm{s}$ для металлов), GDOES показывает хорошее пространственное разрешение по глубине и элементную чувствительность [20]. Демонстрация возможностей GDOES при анализе диэлектрических слоев была проведена при исследовании многослойных оптических покрытий $\mathrm{ZrO}_{x} / \mathrm{SiO}_{x}$ и $\mathrm{TaO}_{x} / \mathrm{SiO}_{x}, \mathrm{SiO}_{2} / \mathrm{TiO}_{2}$ и $\mathrm{SiO}_{2} / \mathrm{Si}_{3} \mathrm{~N}_{4}$ с толщиной каждого слоя около $100 \mathrm{~nm}$ [21].

Обладая схожими характеристиками с другими методами анализа поверхности [22], стоимость приборной реализации метода GDOES и затраты на его обслуживание существенно ниже, чем для других методов анализа поверхности.

Несмотря на обилие публикаций о применении метода GDOES для анализа тонких пленок и покрытий из различных материалов, в литературе отсутствует информация по анализу методом GDOES таких сложных систем, как многокомпонентные пленки ферритовгранатов и гетероструктур на их основе. Для получения таких структур с заданными параметрами в них вводятся до семи различных элементов. В $[23,24]$ представлены результаты исследования пленок ферритов-гранатов, использующихся в качестве магнитоактивных слоев одномерных магнитофотонных и магнитоплазмонных кристаллов, полученных методом реактивного ионно-луче- 
вого распыления (Bi YIG). Особенностью конструирования таких кристаллов является наличие двух магнитоактивных слоев ферритов-гранатов, последовательно напыленных и в дальнейшем кристаллизованных при высокой температуре. При этом важно понимать, как формируются и что собой представляют внутренние и внешние интерфейсы таких структур. В связи с этим целью настоящей работы было проведение профильного анализа однослойных и двуслойных пленок многокомпонентных ферритов-гранатов, полученных реактивным ионно-лучевым распылением, до и после их кристаллизационного отжига.

\section{1. Методика эксперимента}

Синтез пленок ферритов-гранатов с общей формулой $(\mathrm{BiLuGd})_{3}(\mathrm{FeAl})_{5} \mathrm{O}_{12}$ проводили методом реактивного ионно-лучевого распыления в смеси инертного (аргон) и реактивного (кислород) газов [25].

Мишени для ионного распыления готовили по стандартной керамической технологии, включающей прессование исходной смеси окислов, отжиг при $800{ }^{\circ} \mathrm{C}$, помол и повторное прессование с последующим твердофазным синтезом в течение $12 \mathrm{~h}$ при температурах $850^{\circ} \mathrm{C}$ на воздухе при атмосферном давлении.

Были синтезированы мишени двух составов: висмут-замещенные ферриты-гранаты $\mathrm{Bi}_{1.0} \mathrm{Lu}_{0.5} \mathrm{Gd}_{1.5} \mathrm{Fe}_{4.2} \mathrm{Al}_{0.8} \mathrm{O}_{12}$ (Г1) и $\mathrm{Bi}_{2.5} \mathrm{Gd}_{0.5} \mathrm{Fe}_{3.8} \mathrm{Al}_{1.2} \mathrm{O}_{12}$ (Г8). Микроанализ химического состава на растровом электронном микроскопе РЭМ 100 показал близость состава мишеней расчетному составу шихты с некоторым уменьшением содержания висмута после твердофазного синтеза. Рентгенофазовый анализ синтезированных мишеней показал наряду с гранатовой фазой наличие рефлексов фаз феррата висмута $\mathrm{Bi}_{2} \mathrm{Fe}_{4} \mathrm{O}_{9}$ и феррита висмута $\mathrm{BiFeO}_{3}$.

Профильный анализ синтезированных пленок проводился на спектрометре Profiler 2 (Horiba Jobin Yvon S.A.S, Франция), работающем в радиочастотном $(13.56 \mathrm{MHz})$ и импульсном ВЧ-режимах. Длины волн для измеренных линий составляли $(\mathrm{nm})$ : $\mathrm{Bi}-306.772$; $\mathrm{Lu}$ - 261.542; Gd - 342.264; Fe — 371.994; Al 396.152; Ga - 294.364; O - 130.217. Однозначной идентификации элементов в эмиссионном анализе иногда мешает наложение спектральных линий отдельных элементов. Действительно, оно наблюдается для таких пар редкоземельных элементов, как $\mathrm{Y}-\mathrm{Gd}, \mathrm{Sm}-\mathrm{Gd}$, однако $\mathrm{Y}$ и $\mathrm{Sm}$ не входили в состав исследованных пленок. Линзы переходной оптики, формирующие изображение плазмы на входной щели спектрометра, тщательно контролировались. Профиль эмиссионного кратера измерялся на профилометре Mitutoyo SJ 410.

Исследованы аморфные и кристаллизованные однослойные пленки составов ГГГ/Г1 и ГГГ/Г8, а также двуслойные аморфные и кристаллизованные структуры ГГГ/Г1/Г8 и ГГГ/Г8/Г1, осажденные на подложки гадолиний-галлиевого граната $\mathrm{Gd}_{3} \mathrm{Ga}_{5} \mathrm{O}_{12}$ (ГГГ). Толщина слоев Г1 и Г8 составляла $100-150 \mathrm{~nm}$, толщина гетероструктур ГГГ/Г1/Г8 и ГГГ/Г8/Г1 составляла около $200 \mathrm{~nm}$.

\section{2. Результаты исследований и их обсуждение}

Свеженапыленные (не отожженные) пленки ферритов-гранатов аморфны и не обладают магнитными свойствами. Для придания пленкам магнитных свойств их подвергали кристаллизационному отжигу в атмосфере. Динамика процессов кристаллизации исследовалась по возникновению и росту величины эффекта Фарадея, изменению параметров петли магнитного гистерезиса и данным ФМР-спектроскопии [25]. Были определены оптимальные для каждого состава температуры и время отжига: для состава с меньшим содержанием Вi (Г1) это $700{ }^{\circ} \mathrm{C}$ и $20 \mathrm{~min}$, для состава с большим содержанием $\mathrm{Bi}$ (Г8) $-680^{\circ} \mathrm{C}$ и $20 \mathrm{~min}$.

Для удобства восприятия интенсивность линии некоторых элементов (рис. 2-5) при необходимости умножалась (или делилась) на константу (рядом с каждым элементом указан множитель). Таким образом, мы получаем профиль распределения элементов в поверхностных и глубинных слоях, включая элементы подложки.

Важным элементом проведения профильного анализа является выбор таких технологических условий, при которых реализуется максимально однородный профиль травления. На рис. 1 представлен типичный для всех исследованных образцов профиль кратера кристаллизованной пленки. Видно, что дно кратера не идеально плоское, изменение по глубине может составлять до $10 \%$.

Результаты измерения интенсивности спектральных линий от времени ионного травления однослойных аморфных и кристаллизованных пленок составов Г1 и Г8 приведены соответственно на рис. 2 и 3 . Из рисунков видно, что Profiler 2 уверенно фиксирует наличие всех элементов, а также их градиентное распределение на внешнем и внутреннем интерфейсах. В ряде случаев на поверхности фиксировалась линия углерода, связанная

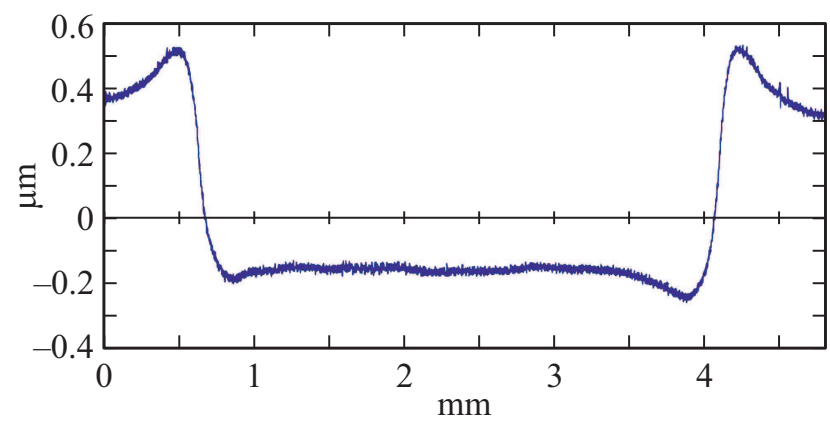

Рис. 1. Типичный для всех исследованных образцов профиль кратера кристаллизованной пленки. 

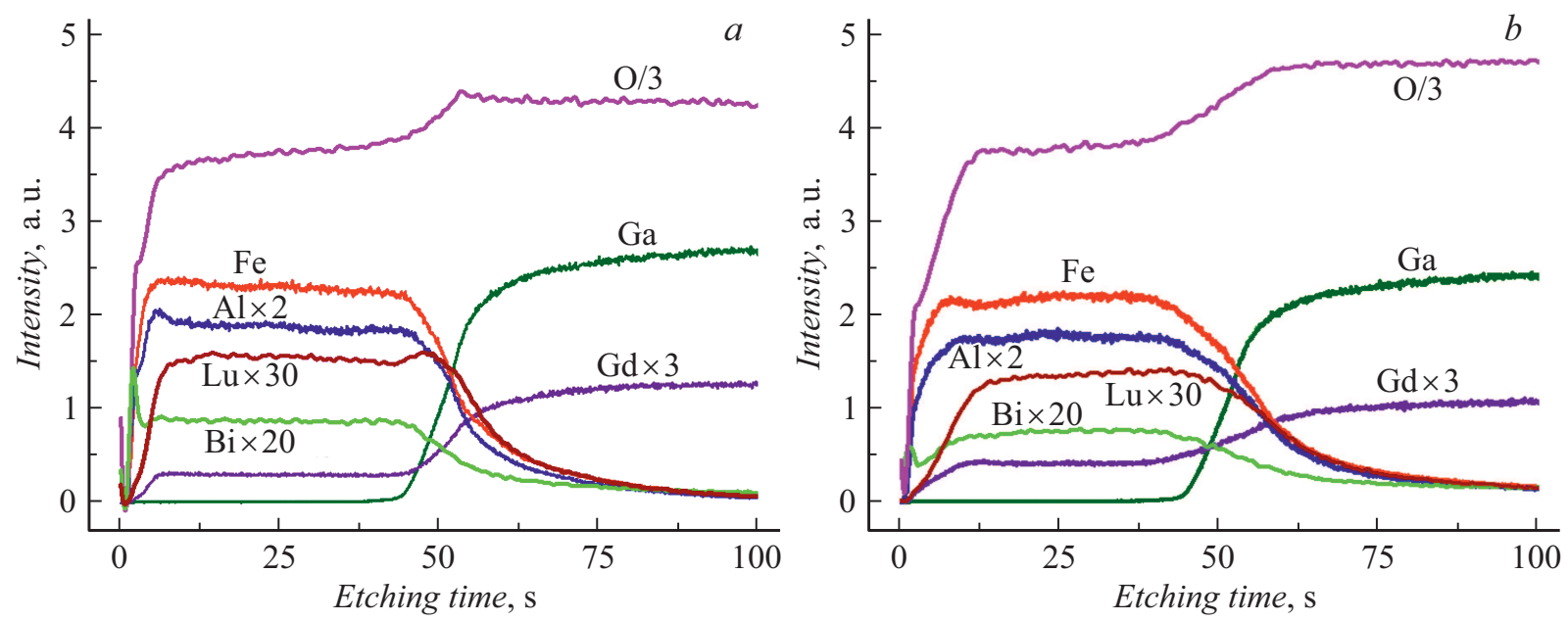

Рис. 2. Профиль элементного состава по толщине аморфной $(a)$ и кристаллизованной $(b)$ при $700{ }^{\circ} \mathrm{C}$ пленок в течение 20 min в структуре Г1 на подложке ГГГ.
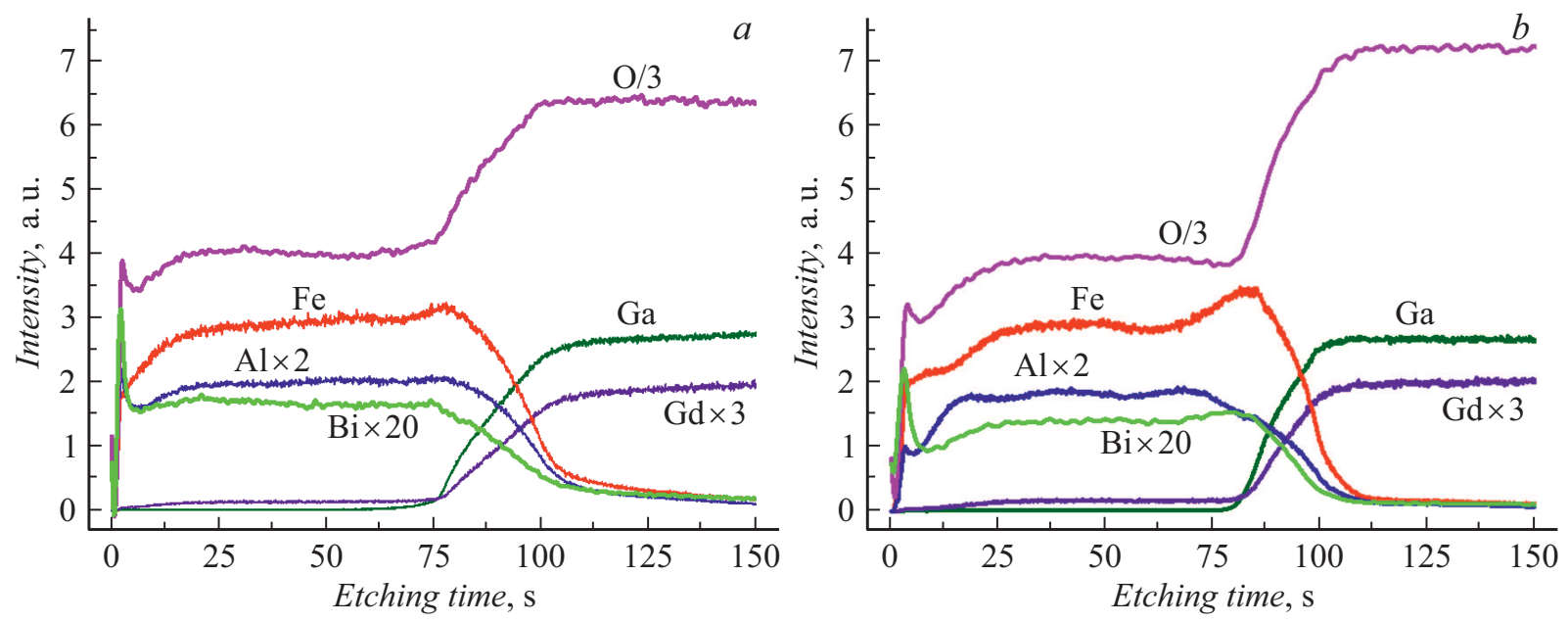

Рис. 3. Профиль элементного состава по толщине аморфной $(a)$ и кристаллизованной $(b)$ при $680{ }^{\circ} \mathrm{C}$ пленок в течение 20 min в структуре Г8 на подложке ГГГ.

с наличием поверхностных загрязнений, которая не представлена на рисунках.

Интерфейсы пленки Г8 до и после кристаллизационного отжига (рис. 3) свидетельствует о том, что внешний интерфейс пленки обогащен атомами висмута и кислорода, причем концентрация Вi на поверхности пленки Г8 в 2.5 раза выше, чем в пленке с Г1 (рис. 2), что полностью коррелирует с его содержанием в пленках. После кристаллизационного отжига в пленке с наименьшим содержание $\mathrm{Bi}$ он практически исчезает в $Г 1$ (рис. 2,b), а в пленке Г8 уменьшается на $30 \%$ (рис. $3, b$ ). Избыток атомов Ві и О может свидетельствовать о присутствии на внешнем интерфейсе нанометровой пленки ортоферрита висмута или оксида висмута. После отжига концентрация всех элементов в пределах 10\% сохраняется. Основные изменения касаются размеров внешних (IFA) и внутренних интерфейсов пленка-пленка (IFF) и пленка-подложка (IFS), которые уширяются после кристаллизации.

Особенностью исследуемых пленок и гетероструктур на их основе является значительное уменьшение концентрации кислорода относительно его концентрации в подложке. Так, в пленке Г1/ГГГ после кристаллизации эта разница составляет около $17 \%$ (рис. 3), а в пленке Г8/ГГГ - 43\% (рис. 3). Поскольку подложка и пленки имеют одну и ту же кристаллическую структуру граната, было неясным сохраняется ли стехиометрия при синтезе гетероструктур. С этой целью был проведен синтез и исследование структур с инвертированными слоями ГГГ/Г1/Г8 и ГГГ/Г8/Г1. На рис. 4 и 5 представлен профиль таких структур до и после кристаллизации. Видно, что независимо от расположения слоев в структуре аномальное соотношение интенсивностей спектральных линий кислорода сохраняется. 

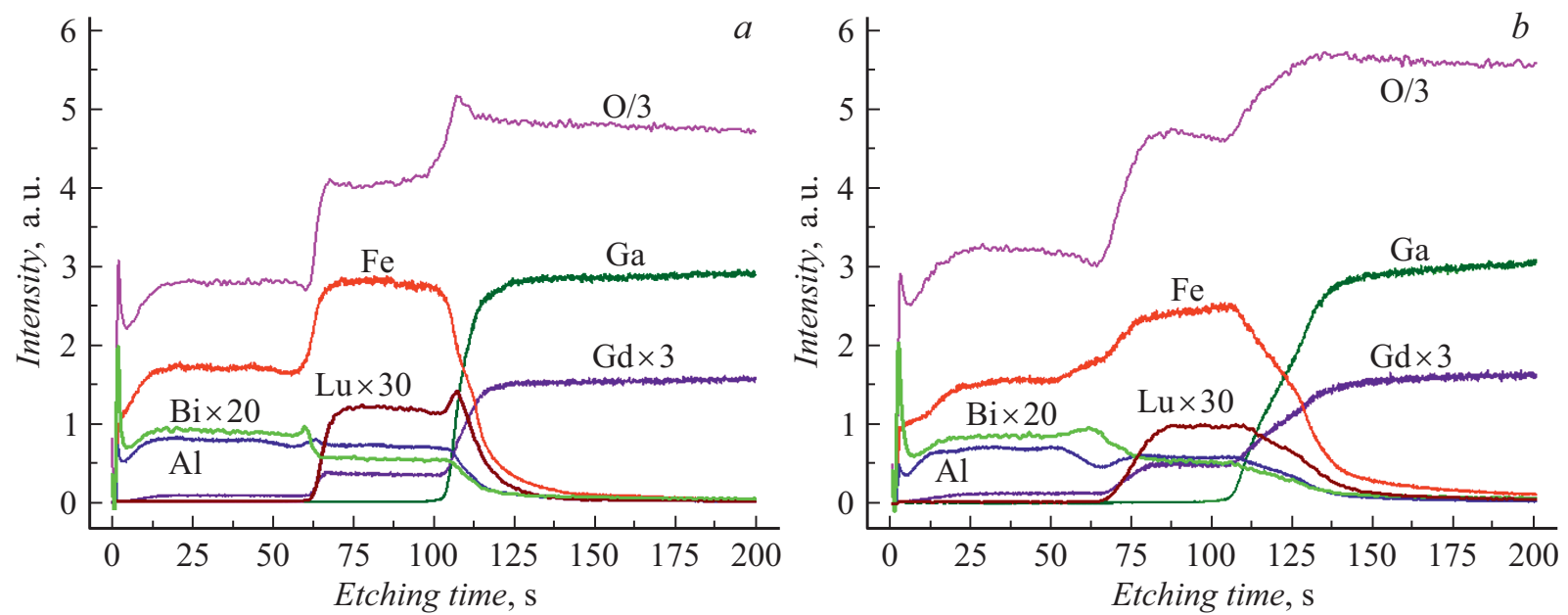

Рис. 4. Профиль элементного состава по толщине аморфной $(a)$ и кристаллизованной $(b)$ пленок в течение 20 min в $\Gamma 1\left(700{ }^{\circ} \mathrm{C}\right)$ и пленок Г8 $\left(680^{\circ} \mathrm{C}\right)$ в структуре ГГГ/Г1/Г8.
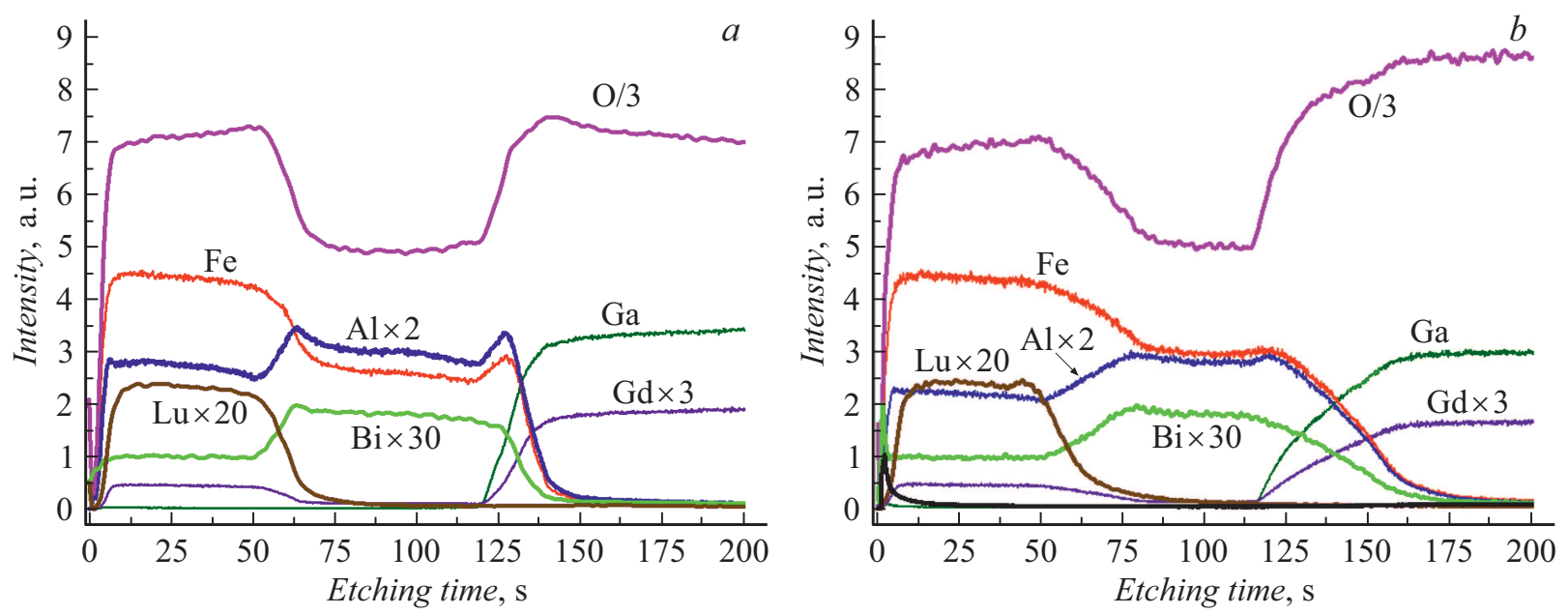

Рис. 5. Профиль элементного состава по толщине аморфной $(a)$ и кристаллизованной $(b)$ пленок в течение 20 min в $Г 8\left(680{ }^{\circ} \mathrm{C}\right)$ и пленок $Г 1\left(700^{\circ} \mathrm{C}\right)$ в структуре ГГГ/Г8/Г1.

Для определения степени стехиометричности синтезированных структур интенсивности спектральных линий каждого элемента $I_{\mathrm{el}}$ были пронормированы на интен-

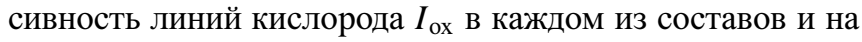
формульное содержание каждого элемента этом составе. Предварительно интенсивности кислородных линий $I_{\text {ох }}$ в каждом составе были пронормированы на интенсивности линий кислорода в подложке $I_{\text {ох }}$ (ГГГ). В таблице представлены нормированные значения интенсивностей всех спектральных линий в отдельных компонентах гетероструктур при различной последовательности их синтеза. Видно, что нормированные интенсивности большинства элементов, включая кислород, независимо от их расположения в гетероструктуре имеют одинаковые или близкие значения. Это означает, что их интенсивность уменьшается синхронно с интенсивностью кислорода, и в основе эффекта аномального снижения интенсивности кислорода в пленках по сравнению с интенсивностью кислорода в подложке может лежать различие в скоростях распыления монокристаллической подложки ГГГ и осажденных на него поликристаллических пленок ферритов на основе висмута. Эти данные также показывают, что в целом стехиометрия ферритовых слоев не зависит от их расположения в гетероструктуре.

Кристаллизационный отжиг приводит к уширению как внешних (IFA), так и внутренних пленка-пленка (IFF) и пленка-подложка (IFS) интерфейсов. Так, например, как видно из рис. 3 , внешний интерфейс в пленках Г8/ГГГ увеличился почти на $50 \%$. Подобным образом ведут себя внешние интерфейсы и в гетероструктурах. Значительно более сильные изменения происходят на внутренних интерфейсах. При кристаллизации структуры ГГГ/Г1/Г8 среднее значение ширины для всех элементов на интерфейсах IFF и IFS увеличились в 2 раза, а в структуре ГГГ/Г8/Г1 ширина интерфейса IFS выросла в 3 раза (рис. 4 и 5). Наибольший вклад в это увеличение внесли 
Относительные значения интенсивностей спектральных линий элементов гетероструктур ГГГ/ Г1/Г8 и ГГГ/ Г8/Г1

\begin{tabular}{c|c|c|c|c}
\hline \multirow{2}{*}{ Элемент } & \multicolumn{2}{|c|}{$\mathrm{Bi}_{1.0} \mathrm{Lu}_{0.5} \mathrm{Gd}_{1.5} \mathrm{Fe}_{4.2} \mathrm{Al}_{0.8} \mathrm{O}_{12}(\Gamma 1)$} & \multicolumn{2}{c}{$\mathrm{Bi}_{2.5} \mathrm{Gd}_{0.5} \mathrm{Fe}_{3.8} \mathrm{Al}_{1.2} \mathrm{O}_{12}(\Gamma 8)$} \\
\cline { 2 - 5 } & $\Gamma 1 / \Gamma 8$ & $\Gamma 8 / \Gamma 1$ & $\Gamma 1 / \Gamma 8$ & $\Gamma 8 / \Gamma 1$ \\
\hline $\mathrm{O}$ & 7.22 & 7.32 & 5.11 & 5.22 \\
\hline $\mathrm{Fe}$ & 4.34 & 5.41 & 0.13 & 5.23 \\
\hline $\mathrm{Bi}$ & 0.22 & 0.23 & 0.62 & 0.21 \\
\hline $\mathrm{Gd}$ & 0.73 & 0.44 & 6.23 & 0.41 \\
\hline $\mathrm{Al}$ & 5.13 & 6.31 & - & 7.44 \\
\hline $\mathrm{Lu}$ & 0.61 & 1.23 & & -
\end{tabular}

такие элементы, как $\mathrm{Fe}, \mathrm{Al}$ и $\mathrm{Bi}$. Отметим, что в то время как интерфейс IFF формируется только элементами феррит-гранатовых пленок, интерфейс IFS формируется в результате взаимной диффузии элементов феррита и элементов подложки ГГГ. Увеличение ширин интерфейсов при отжиге связано с взаимодиффузией гранатообразующих элементов на границах интерфейсов. Благодаря размытию внутреннего IFF интерфейса отдельные компоненты гетероструктуры являются обменно-связанными, что подтверждается отсутствием слоистости в магнитооптических петлях гистерезиса и в спектрах ферромагнитного резонанса [23,24].

\section{Заключение}

Для всех исследованных феррит-гранатовых наноструктур можно отметить следующие особенности профильного распределения элементов. В пленках и гетероструктурах на их основе существуют несколько интерфейсных областей, распределение элементов в которых отличается от распределения в объеме. Размеры внешних интерфейсов значительно меньше внутренних, они обогащены атомами висмута, концентрация которого растет пропорционально его содержанию в составе пленок. Размеры всех интерфейсных областей в кристаллизованных пленках шире, чем в исходных пленках с аморфной структурой. Под действием кристаллизационного отжига избыток висмута существенно уменьшается. Кислород по толщине аморфных и кристаллизованных пленок и структур изменяется ступенями с шагом, соответствующим числу интерфейсов. Показано, что, несмотря на подобную аномалию, стехиометрия составов в каждом из слоев сохраняется. Это означает, что метод оптической эмиссионной спектроскопии тлеющего разряда дает адекватную картину распределения элементов и применим для послойного анализа таких сложных диэлектрических наноструктур, как структуры на основе ферритов-гранатов.

Результаты, полученные в ходе экспериментов по профильному анализу в наноразмерных структурах ферритов-гранатов, характеризуют диффузионные процессы, происходящие в процессе кристаллизации, и могут быть использованы для корректировки технологических режимов (времени, скорости отжига и температуры) с целью получения пленок и структур с заданными характеристиками.

\section{Финансирование работы}

Исследование выполнено при поддержке гранта Российского научного фонда (проект № 19-72-20154).

\section{Конфликт интересов}

Авторы заявляют, что у них нет конфликта интересов.

\section{Список литературы}

[1] T. Nelis, R. Payling. Glow Discharge Optical Emission Spectroscopy: A Practical Guide (Royal Society of Chemistry, Cambridge, 2003). DOI: 10.1039/9781847550989.

[2] A. Bengtson. Spectrochim. Acta, 49B (4), 411 (1994). DOI: $10.1016 / 0584-8547(94) 80034-0$

[3] М. Нехин, А. Кузнецов, П. Шапон. Аналитика, 5 (4), 34 (2012).

[4] Ф.В. Кирюханцев-Корнеев. Известия вузов. Порошковая металлургия и функциональные покрытия, 2, 60 (2013). DOI: $10.17073 / 1997-308 X-2013-2-60-70$

[5] J.M. Long. Proc. of the 10th Asia-Pacific-Conf. on NonDestructive Testing (Brisbane, Australia, 2001) http://www.ndt.net/article/apcndt01/

[6] D.V. Shtansky, A.V. Bondarev, Ph.V. Kiryukhantsev-Korneev, A.N. Sheveyko, Yu.S. Pogozhev. Surf. Coat. Technol., 206, 2506 (2012). DOI: 10.1016/j.surfcoat.2011.10.057

[7] E. Levashov, M. Petrzhik, M. Tyurina, F. KiryukhantsevKorneev, P. Tsygankov, A. Rogachev. Metallurgist, 54 (9-10), 623 (2011). DOI: 10.1007/s11015-011-9350-5

[8] R. Grieseler, T. Kups, M. Wilke, M. Hopfeld, P. Schaaf. Mater. Lett., 82, 74 (2012). DOI: 10.1016/j.matlet.2012.05.055

[9] T. Polcar, R. Martinez, T. Vítů, L. Kopecký, R. Rodriguez, A. Cavaleiro. Surf. Coat. Technol., 203, 3254 (2009). DOI: $10.1016 /$ j.surfcoat.2009.04.005 
[10] S. Fouvry, B. Wendler, T. Liskiewicz, M. Dudek, L. Kolodziejczyk. Wear, 257 (7-8), 641 (2004). DOI: $10.1016 /$ j.wear.2004.02.009

[11] T. Nelis, J. Pallosi. Appl. Spectrosc. Rev., 41 (3), 227 (2006). DOI: $10.1080 / 05704920600620345$

[12] R. Escobar Galindo, E. Forniís, J.M. Albella. J. Anal. At. Spectrom., 20, 1108 (2005). DOI: 10.1039/B502771C

[13] M. Wilkeac, G. Teicherta, R. Gemmab, A. Pundtb, R. Kirchheimb, H. Romanusc, P. Schaafac. Thin Solid Films, 520 (5), 1660 (2011). DOI: 10.1016/j.tsf.2011.07.058

[14] H. Habazaki, M. Uemura, K. Fushimi, E. Tsuji, Y. Aoki, S. Nagata. J. Surf. Finish. Soc. Jpn., 66 (12), 670 (2015). DOI: $10.4139 /$ sfj.66.670

[15] А.Е. Кудряшов, Е.И. Замулаева, Е.А. Левашов, Ф.В. Кирюханцев-Корнеев, А.Н. Шевейко, Н.В. Швындина. Электронная обработка материалов, 55 (2), 10 (2019). DOI: $10.5281 /$ zenodo.2629552

[16] K. Shimizu, H. Habazaki, P. Skeldon, G.E. Thompson. Surf. Interface Anal., 29 (12), 887 (2000). DOI: 10.1002/10969918(200012)29:12<887::AID-SIA942>3.0.CO;2-X

[17] K. Shimizu, H. Habazaki, P. Skeldon, G.E. Thompson, G.C. Wood. Surf. Interface Anal., 29(2), 151 (2000). DOI: $\quad 10.1002 /($ SICI $) 1096-9918(200002) 29: 2<151:: A I D-S I A$ 728>3.0.CO;2-V

[18] K. Shimizu, R. Payling, H. Habazaki, P. Skeldon, G.E. Thompson. J. Anal. At. Spectrom., 19, 692 (2004). DOI: 10.1039/B400918P

[19] B. Ferná ndez, N. Bordel, R. Pereiro, A. Sanz-Medel. J. Anal. At. Spectrom., 18, 151 (2003). DOI: 10.1039/B211422D

[20] P. Le Coustumer, M. Motelica-Heino, P. Chapon, H. Francois Saint-Cyr, R. Payling. Surf. Interface Anal., 35 (7), 623 (2003). DOI: $10.1002 /$ sia. 1584

[21] V.-D. Hodoroaba, W.E.S. Unger, H. Jenett, V. Hoffmann, B. Hagenhoff, S. Kayser, K. Wetzig. Appl. Surf. Sci., 179 (1-4), 30 (2001). DOI: 10.1016/S0169-4332(01)00259-8

[22] S. Suzuki, K. Kakita. J. Surf. Anal., 12 (2), 174 (2005).

[23] V.N. Berzhansky, T.V. Mikhailova, A.V. Karavainikov, A.R. Prokopov, A.N. Shaposhnikov, I.N. Lukienko, Yu.N. Kharchenko, O.V. Miloslavskaya, N.F. Kharchenko. J. Magn. Soc. Jpn., 36 (1-2), 42 (2012).

DOI: $10.3379 / \mathrm{msjmag} .1108 \mathrm{M} 006$

[24] E.Yu. Semuk, V.N. Berzhansky, A.R. Prokopov, A.N. Shaposhnikov, A.V. Karavainikov, O.Yu. Salyuk, V.O. Golub. J. Magn. Magn. Mater., 394, 92 (2015). DOI: $10.1016 /$ j.jmmm.2015.06.047

[25] A.N. Shaposhnikov, A.V. Karavainikov, A.R. Prokopov, V.N. Berzhansky, O.Y. Salyuk. Mater. Res. Bull., 47, 1407 (2012). DOI: 10.1016/j.materresbull.2012.02.049 